\title{
SPECIFIC INFLUENCE OF STRIATAL TARGET NEURONS ON THE IN VITRO OUTGROWTH OF MESENCEPHALIC DOPAMINERGIC NEURITES: A MORPHOLOGICAL QUANTITATIVE STUDY ${ }^{1}$
}

\author{
SUZANNE DENIS-DONINI, ${ }^{2}$ JACQUES GLOWINSKI, ${ }^{3}$ AND ALAIN PROCHIANTZ
}

Groupe NB, Institut National de la Santé et de la Recherche Médicale U.114, Collège de France, 11 place Marcelin Berthelot, 75231 Paris Cedex 5, France

Received December 20, 1982; Revised May 20, 1983; Accepted May 20, 1983

\begin{abstract}
In previous studies, we have shown that dissociated dopaminergic neurons from embryonic mouse in co-culture with striatal target neurons take up and synthesize dopamine to a greater extent. We now report that striatal target cells influence the morphology of dopaminergic neurons as well. In co-culture, the total length of neuritic arborization visualized by autoradiography is reduced when compared to cultures of mesencephalic neurons alone or to co-cultures with cerebellar cells. Experiments performed in the presence of striatal glial cells at the same density as striatal neurons or with mcdia conditioned with striatal glia or neurons suggest that striatal neurons regulate dopaminergic afferent growth in vitro through specific neuro-neuronal interactions.
\end{abstract}

The nigrostriatal dopaminergic (DA) pathway has been used for several years in our laboratory as a model for studying the role of neuronal interactions in the development of central neurons. Indeed, mesencephalic DA neurons of mouse embryos can be dissociated and cultured either in the absence or in the presence of their target cells from the striatum. These DA neurons were shown to develop biochemical and morphological characteristics in vitro similar to those found in vivo (Prochiantz et al., 1979; Berger et al., 1982). Interestingly, their ability to take up and synthesize $\left[{ }^{3} \mathrm{H}\right]$ dopamine was increased in the presence of striatal cells, revealing a stimulatory effect of the target on the expression of DA properties. Moreover, similar results were obtained under culture conditions which limit the number of non-neuronal cells to less than $5 \%$ of the total cell population (Di Porzio et al., 1980; Prochiantz et al., 1982). This indicated that neuro-neuronal interactions occur between mesencephalic DA neurons and striatal target cells in vitro. More recently, a similar stimulatory phenomenon was observed when striatal cells were replaced by striatal membranes from young animals (Prochiantz et al., 1981). The present study was undertaken to deter-

\footnotetext{
${ }^{\prime}$ S. D. -D. is a recipient of a long-term European Molecular Biology Organization fellowship. This work was supported by Institut National de la Santé et de la Recherche Médicale, Rhône-Poulenc S. A., and MRI Grant 82.E.0125.

${ }^{2}$ Present address: Laboratory of Molecular Embryology, Arco Felice, Naples, Italy.

${ }^{3}$ To whom correspondence should be addressed.
}

mine whether such biochemical changes are associated with morphological modifications of the DA neurons. For this purpose, mesencephalic DA neurons of mouse embryos were grown under several culture conditions and the total neurite length was analyzed quantitatively, the DA neurons being identified by autoradiography after incubation of the cells with $\left[{ }^{3} \mathrm{H}\right]$ dopamine. In contrast, with predictions which could have been made from biochemical data, we show that the processes of the DA cells are significantly shorter when the neurons are grown in the presence of their striatal target cells than when cultured alone. In fact, it appears that DA growth cones have a tendency to stop when they reach one or more cells. Conversely, growth cones of DA neurons grown in the presence of non-target cells, such as nonDA mesencephalic cells or cerebellar cells, do not establish such frequent contacts, and their processes terminate in an apparently random fashion over the substrate.

\section{Materials and Methods}

Culture conditions. The rostral mesencephalon and the striatum from 13- and 15-day-old Swiss mouse embryos, respectively (Iffa Credo, France), were dissociated in a medium consisting of minimum essential medium and nutrient mixture F-12 (1:1, GIBCO) enriched with glucose $\left(3.3 \times 10^{-2} \mathrm{M}\right)$, glutamine $\left(2 \times 10^{-3} \mathrm{M}\right)$, and sodium bicarbonate $\left(0.3 \times 10^{2} \mathrm{M}\right)$. Unless specifically stated, mesencephalic cells $\left(5 \times 10^{5}\right)$ or mesencephalic plus striatal cells $\left(2.5 \times 10^{5} \mathrm{cach}\right)$ were plated on polyornithine $\left(1.5 \mu \mathrm{g} / \mathrm{ml}, M_{r}=40,000\right.$, Sigma Chemical Co. - 
coated culture dishes $\left(9.5 \mathrm{~cm}^{2}\right.$, Falcon). The cultures were usually grown in complete medium with $10 \%$ heatinactivated fetal calf serum (Serumaid). Cells were examined after 2, 4, and 6 days without medium changes. In some cases, chemically defined serum-free media were used in which serum was replaced by a mixture of proteins, hormones, and salts. Under these conditions it was shown previously that non-neuronal elements did not represent more than $5 \%$ of the total cell population (Di Porzio et al., 1980; Prochiantz et al., 1982).

For co-cultures with added glial cells, striatal cells from 15-day-old embryos were plated at high density $\left(2 \times 10^{6}\right.$ cells/dish). After 7 days the dishes were washed several times with fresh medium and the cells were grown for 3 weeks, the medium being changed twice a week. At this time most neurons had degenerated and the glial cells formed several confluent layers. The absence of neurons was checked by incubations with an antibody against the $M_{r}=70,000$ neurofilament protein (a kind gift of Dr. D. Paulin). The astroglial nature of the cells was demonstrated by their ability to be decorated with an antibody against glial fibrillary acidic protein (GFAP). These cells were removed from the dish by a brief treatment ( 15 min) with Viokase $0.1 \%$ (GIBCO) in $\mathrm{Ca}^{2+}$ - and $\mathrm{Mg}^{2+}$ free phosphate-buffered saline (PBS), dissociated by gentle pipetting, and replated with freshly prepared mesencephalic cells $\left(2.5 \times 10^{5}\right.$ each).

The conditioned medium from glial cells was obtained by incubating the confluent monolayers with serumcomplete medium for 3 days. This medium was centrifuged at $500 \times g$, filtered, and used in equal proportion with fresh medium. The conditioned medium from neuronal cells was obtained by the same procedure except that glial cells were replaced by striatal neurons previously grown for 1 week in chemically defined serum-free medium.

Autoradiography. The cultures were incubated for $1 \mathrm{hr}$ at $37^{\circ} \mathrm{C}$ with $\left.~^{3} \mathrm{H}\right]$ dopamine $\left(5 \times 10^{-7} \mathrm{M}, 9.9 \mathrm{Ci} / \mathrm{mmol}^{-1}\right.$, New England Nuclear) in PBS completed with $\mathrm{Ca}^{2+}$ and $\mathrm{Mg}^{2+}\left(10^{-3} \mathbf{M}\right.$ each). Incubation was ended by three PBS washes and the cells were fixed for $1 \mathrm{hr}$ at room temperature with $1.5 \%$ glutaraldehyde in PBS. After several PBS washes, they were air dried and coated with Ilford K5 liquid emulsion diluted with water (1:1) and exposed for 1 week in the dark at $4^{\circ} \mathrm{C}$ prior to development with Kodak Dektol. We had shown previously that under our conditions the mesencephalic cultures are devoid of noradrenergic neurons and that $\left[{ }^{3} \mathrm{H}\right]$ dopamine is only taken up in DA neurons (Berger et al., 1982).

Neurite measurement. In order to measure the length of the labeled neurons, one neuron out of three was systematically photographed and the total neurite length per neuron was measured using a tracing table. An estimate of the total neurite length was also obtained by counting for each of the 100 first neurons encountered the number of intersections of the processes with all vertical and horizontal lines of a $1-\mathrm{cm}^{2}$ reticle located in the ocular. The distance between two horizontal or vertical lines was $1 \mathrm{~mm}$.

Immunocytochemical studies. Cells after 4 days in coculture were eventually incubated with a monoclonal supernatant against brain surface protein 2 (BPS-2) raised in the rat and provided by Dr. C. Goridis, this BSP-2 protein being identical to N-CAM and known to label only neurons in vitro (Hirn et al., 1981; Chuong et al., 1982). After a $1-\mathrm{hr}$ incubation at $37^{\circ} \mathrm{C}$, the coverslips were rinsed with PBS and incubated with goat anti-rat fluorescein-labeled immunoglobulins ( $1 \mathrm{hr}$ at room temperature). Permeabilization of the cells was achieved with $3.7 \%$ formaldehyde plus $1 \%$ methanol $(20 \mathrm{~min}$ at room temperature), and the incubation was pursued with an antibody against tyrosine hydroxylase. The antigenantibody complex was revealed by a second goat antirabbit fluorescein-conjugated antibody. Fluorescence was examined using a Leitz Orthoplan microscope. The antibody against tyrosine hydroxylase was a kind gift of Dr. A. Vigny.

\section{Results}

Morphological features of the mesencephalic DA cells in the absence and presence of striatal target cells. Dopaminergic neurons in culture with striatal target cells display different morphological features from those grown in the absence of target. Figure 1 illustrates the difference in the aspects of the neurons under both culture conditions after 4 days. In the absence of target, D $\Lambda$ neurons extend long processes that wander about on the substrate and usually end in empty spaces in the dish as well as on cells (Fig. $1 A$ ). In contrast, in the presence of target cells, DA processes appear shorter and most of the time terminate on one or a few other cells (Fig. $1 B$ ). 'The short distance covered by the neurite in co-culture seems to be related to its encountering target cells, and in some cases an apparent spreading over and around the contacted cell is observed (Fig. 1D). However, these contacts do not altogether prevent further elongation of the neurites. Continued growth leading to contacts with additional cells is often observed (Fig. $1 C$ ). This pattern is reminiscent of the known ability of monoaminergic neurons to establish synapses en passage.

Elongation rate of $D A$ neurites in the absence or presence of striatal target cells or cerebellar non-target cells. The previous morphological observations encouraged us to measure the neurites of DA cells under different culture conditions. First, the mesencephalic cells, which include a subpopulation of mesencephalic DA neurons, were grown either alone at a final density of $5 \times 10^{5}$ cells/dish or together with striatal or cerebellar cells. In the latter case (co-cultures), $2.5 \times 10^{5}$ mesencephalic cells were mixed with an equal number of either target (striatal) or non-target (cerebellar) cells. The cells in cultures and co-cultures were grown for 4 days before being processed for autoradiography. The distribution of the length of the neurites obtained in this type of experiment are given in Figure 2. These results indicate that striatal target cells induce a 2-fold significant reduction of the mean length of DA neurites, a phenomenon that is not observed in the presence of cerebellar cells.

Since morphological observations in co-culture with striatal cells (Fig. $1 D$ ) suggest that DA cells are able to resume growth after having established some kind of contact with other cell types, it was of interest to measure the lengths of neurites in culture and in co-culture after various times: 2, 4, and 6 days in vitro. From these 


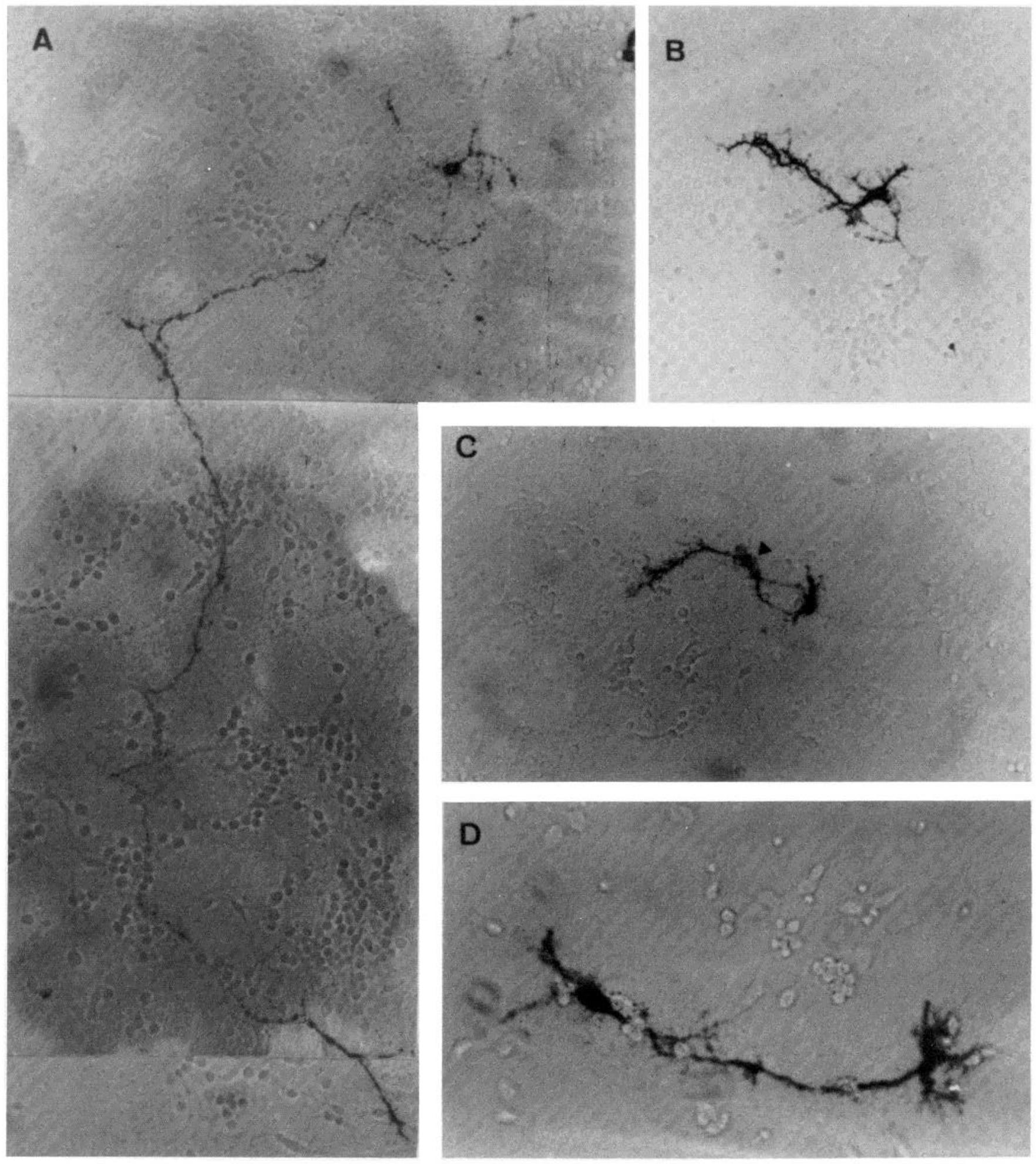

Figure 1. Autoradiography of DA neurons after 4 days in culture. Cells were grown in the absence of target cells $(A)$ or in the presence of striatal cells $(B)$ (Magnification $\times 211)$. Higher magnification $(\times 338)$ of a neuron in co-culture illustrating contacts is shown in $D$. In $C$, the arrowhead indicates the possible position of a first contact and spreading followed by further neuritic growth (Magnification $\times 211$ ).

measurements, an elongation rate of $125 \mu \mathrm{m} /$ day in culture and of $60 \mu \mathrm{m} /$ day in co-culture can be calculated. Figure 3 illustrates the average rate of neurite elongation under either condition. The two curves are parallel and give no indication of a permanent arrest of the DA neurites in the presence of striatal cells. This suggests that there is either a slowing down or a transient arrest of growth in conjunction with cell contact.

Evidence for a role of direct neuro-neuronal interactions in the regulation of DA neurite extension. Besides the 


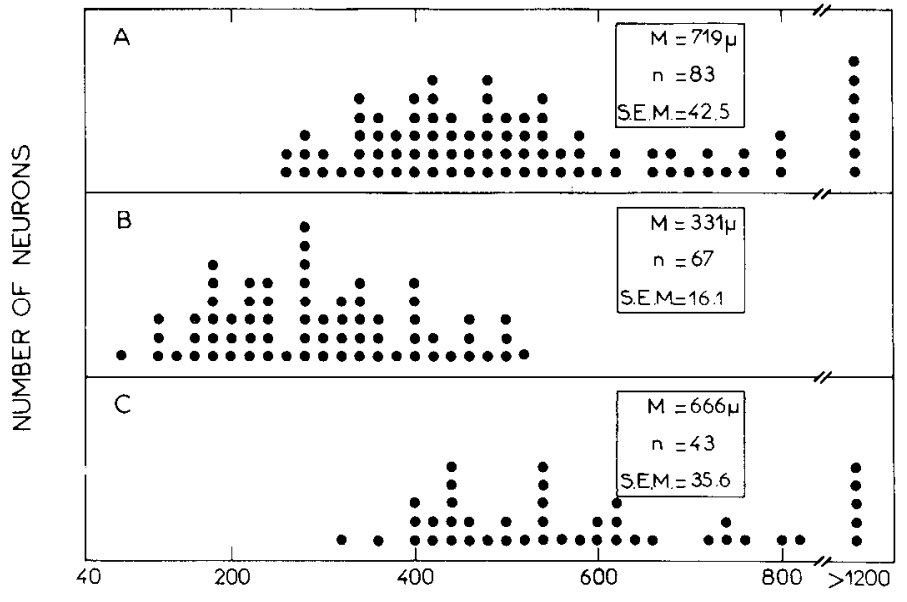

NEURITE LENGTH $(\mu \mathrm{m})$

Figure 2. Length distribution of DA neurites. Dopaminergic neurites were photographed and their total length was measured. The distributions observed in culture $(A)$, in co-culture with striatal cells $(B)$, or with cerebellar cells $(C)$ are presented. Differences in length inferior to $10 \mu \mathrm{m}$ were neglected. The number of neurons analyzed $(n)$, the mean length $(M)$, and the standard error of the mean (S.E.M.) are indicated in each case.

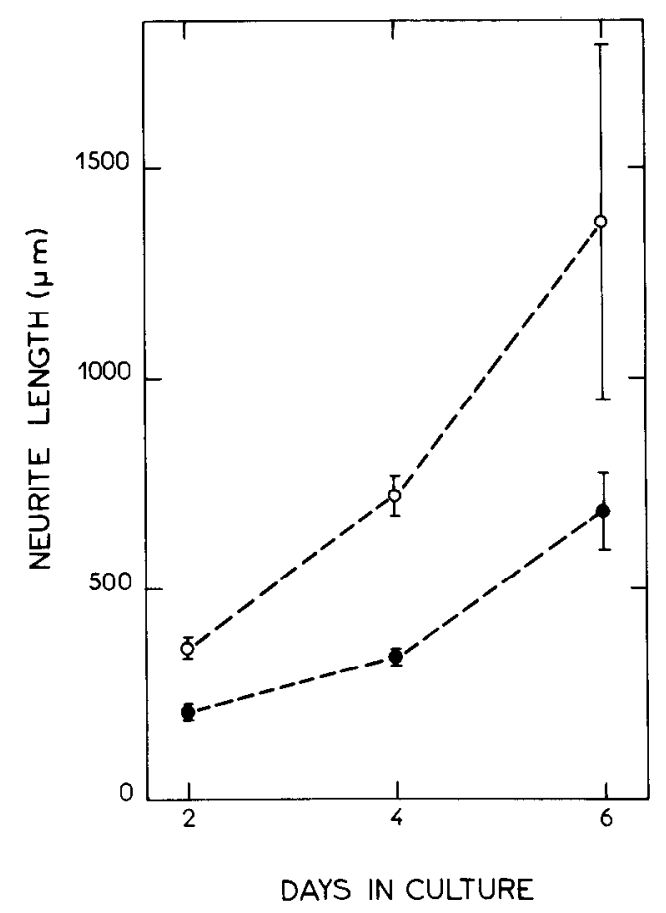

Figure 3. Average rate of DA neurite elongation. The total neurite length was measured for about 100 neurons in each case. The mean length and the standard error of the mean are represented by points and bars. - in the presence of striatal cells; $\mathrm{O}_{-}-\mathrm{O}$, in the absence of striatal cells.

occurrence of cell-cell surface interactions, the fact that mesencephalic DA neurites were shorter in the presence of striatal cells could also result from a dilution of trophic factors released by mesencephalic DA or non-DA cells since their number was reduced by a factor of 2 in coculture $\left(2.5 \times 10^{5}\right.$ versus $5 \times 10^{5}$ cells $)$. The release by striatal cells of factors having an inhibitory effect on DA neurite growth could be another possibility.
In spite of the fact that a dilution effect was not supported by the results obtained in co-culture with cerebellar cells, this hypothesis was tested further. For this purpose, cultures and co-cultures were established at the same final concentration of mesencephalic cells (5 $\times 10^{5} /$ dish). In the co-cultures $5 \times 10^{5}$ striatal cells were added, leading to a final concentration of $10^{6}$ cells/plate. In this experiment and in the following ones, the total length of neurites was estimated by counting the number of intersections with all lines of a micrometer. This was done in each case for about $100 \mathrm{DA}$ neurons taken at random. In spite of an identical number of mesencephalic cells, the mean length of DA neurites was still twice as short in co-culture (Fig. 4). In order to look for an

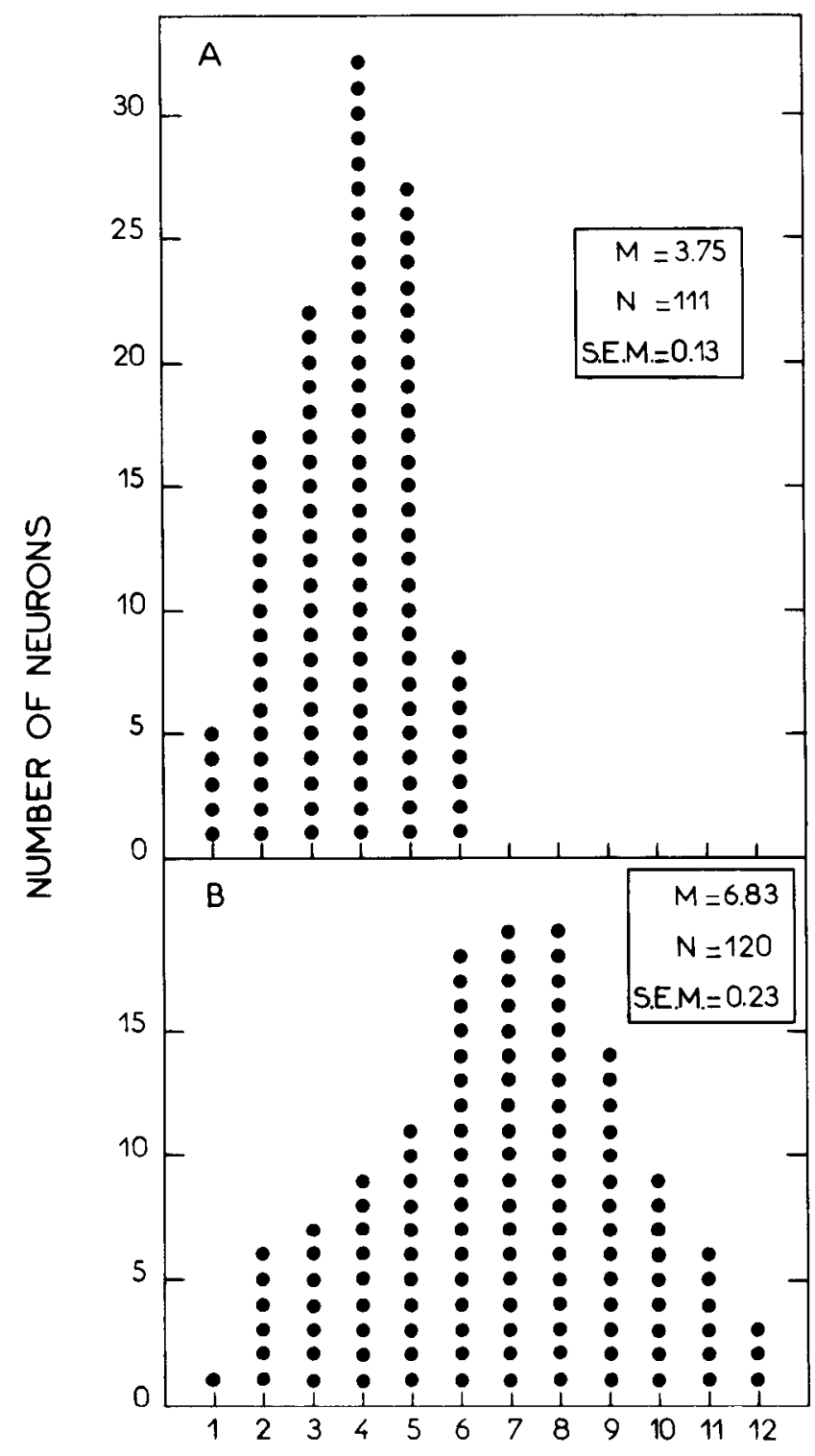

\section{NUMBER OF INTERSECTIONS}

Figure 1. Addition of striatal target cells to mesencephalic neurons. In this case $5 \times 10^{5}$ striatal cells were added to $5 \times$ $10^{5}$ mesencephalic cells $(A)$ and the length distribution of DA neurites was compared to that obtained in the presence of $5 \times$ $10^{5}$ mesencephalic cells only $(B)$. 
eventual striatal inhibitory factor in co-culture, mesencephalic cells were plated in conditioned synthetic media from mesencephalic or striatal neurons. After 2 days in culture, the mean lengths of the DA neurites were similar in both cases but significantly greater $(377 \pm 26 \mu \mathrm{m})$ than those observed in non-conditioned synthetic me$\operatorname{dium}(221 \pm 29 \mu \mathrm{m})$. These results indicate that synthetic media conditioned on neurons improve the culture conditions regardless of the origin of the cells chosen for the conditioning. A similar observation was made when mesencephalic cells were grown in a serum containing medium conditioned on striatal glial cells. All of these experiments suggest that contact with striatal cells is a prerequisite for retarded DA neurite elongation. They do not completely rule out that soluble factors may intervene in view of the possible lability of such factors.

Another point of interest to elucidate in this target effect is the respective roles which could be played by neurons and glial cells, although the cultures were prepared from cells of 13- and 15-day-old embryos, ages at which glial development is in an initial stage. Thus mesencephalic cells were grown in co-culture with striatal glial cells or with a glial-conditioned medium (as control). Glial cells were prepared as described under "Material and Methods." As shown in Figure 5, striatal glia in the absence of striatal neurons did not reduce the elongation rate of mesencephalic DA cell neurites. On the contrary, they exerted a slight stimulatory effect after
2 days in culture (Fig. $5, A$ and $B$ ), but this effect was not observed any longer after 5 days (Fig. 5, $C$ and $D$ ). Although this latter phenomenon should be investigated further, it seems that in our experimental conditions the reduced length of the DA processes observed in coculture with striatal cells is not due to glial cells and that cell contact, in order to be effective, must be with target neurons rather than with glia.

However, the glial cells used in this experiment are much older than the neurons since they have been kept 3 weeks in vitro before passage. In order to better characterize the cellular types with which DA neurons are in contact, cells were incubated with an antibody against BSP-2, an antigen known to be neuron specific in vitro (Hirn et al., 1981; Chuong et al., 1982). In this case DA cells were identified with an antibody against tyrosine hydroxylase. After 4 days in culture, most cells were labeled with this antibody, and tyrosine hydroxylasepositive neurites stopped at the level of BSP-2-decorated neurons (Fig. 7A). Moreover, when co-culture experiments were performed in synthetic medium excluding the survival of more than $5 \%$ of glial cells (Prochiantz et al., 1982), contacts could be observed between DA cells and other cells likely to be neurons (Fig. 6B). These two types of experiments reinforce the possibility of neuroneuronal interactions. Nevertheless, interactions with glial elements could also occur when co-cultures are made in the presence of serum.

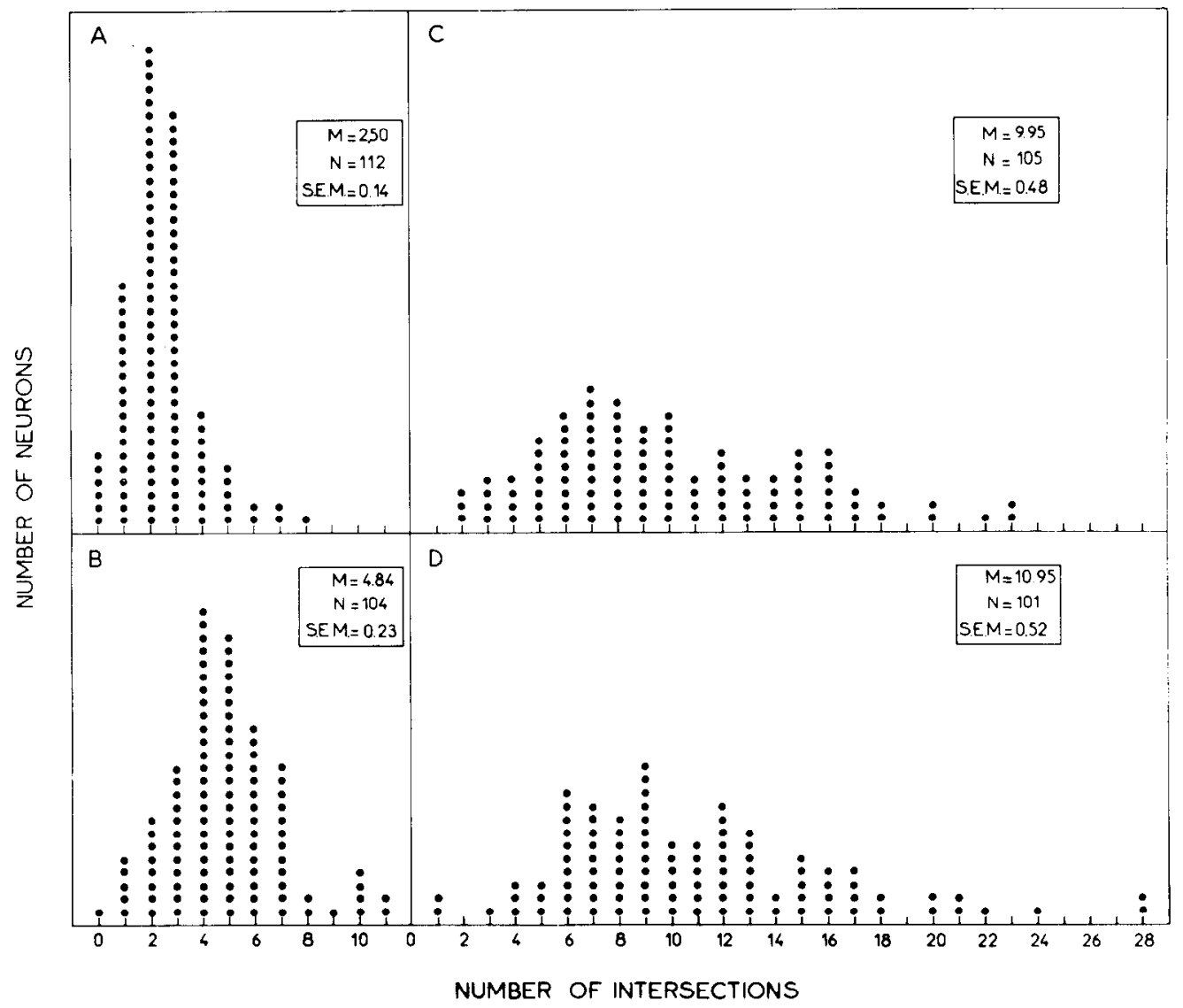

Figure 5. Influence of glial cells on the length of DA neurites. Length distribution of DA neurites after 2 days $(A$ and $B)$ and 5 days $(C$ and $D)$ in the presence of striatal glial conditioned medium ( $A$ and $C$ ) or striatal glial cells ( $B$ and $D$ ). 

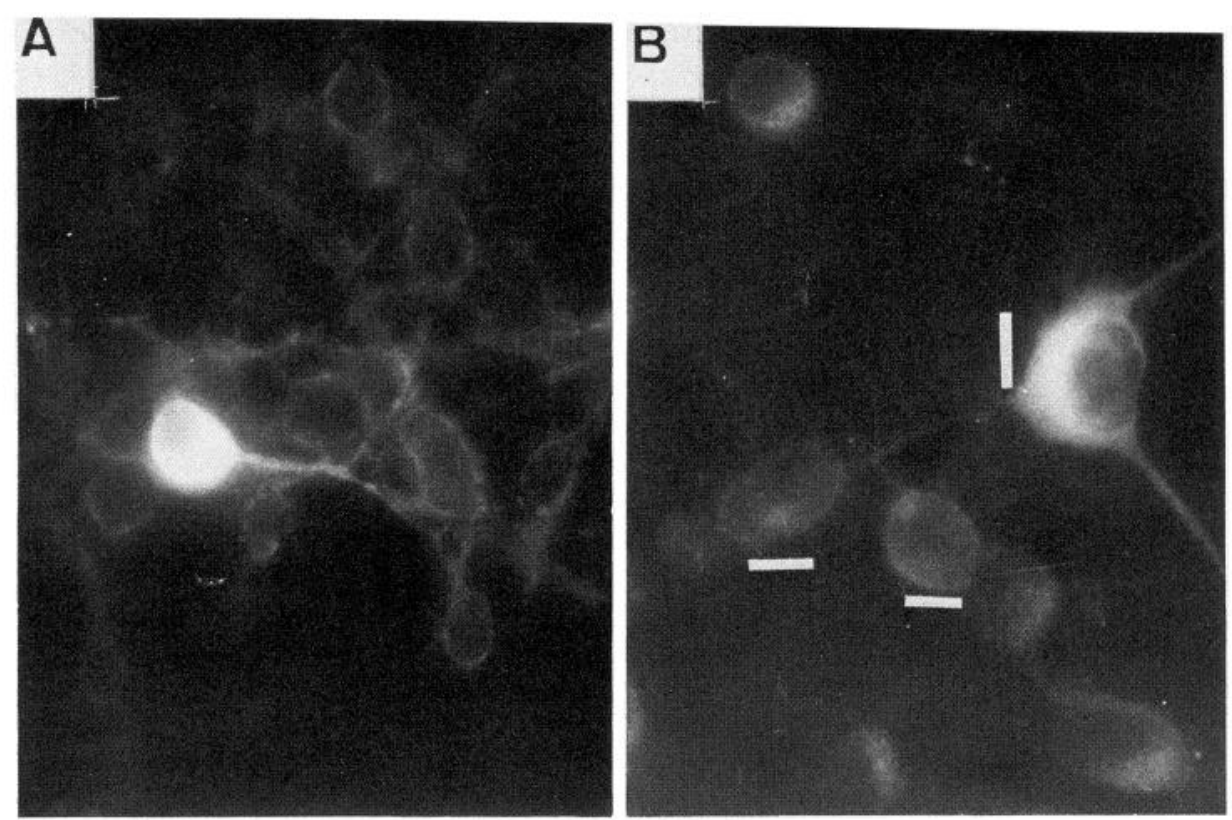

Figure 6. Evidence of neuro-neuronal interactions between DA neurons and other neuronal types in co-culture with striatal cells. A, Cells grown in serum-containing medium were decorated with an antibody against BSP-2 and the DA neuron was visualized using an antiserum against tyrosine hydroxylase. Magnification $\times 400$. $B$, Cells grown in serumfree medium were permeabilized and DA neurons were decorated with an antibody against tyrosine hydroxylase. Two horizontal bars indicate the presence of two cells contacted by the DA neurite emerging from the cell body at the level of the vertical bar. Magnification $\times 612$.

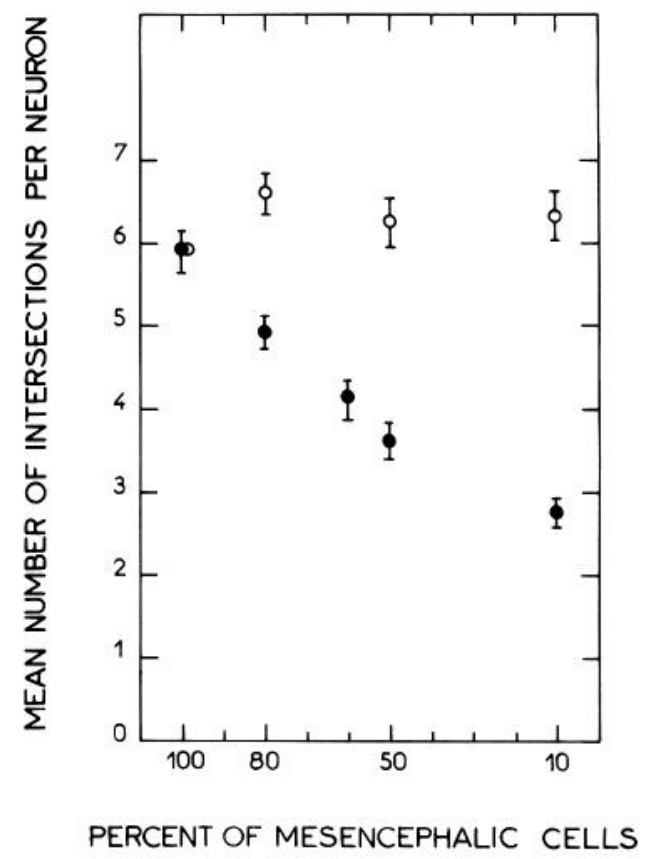

Figure 7. Mean lengths of DA neurites in 4-day co-cultures with increasing amounts of cerebellar or striatal cells. Increasing amounts of striatal $(\Theta)$ or cerebellar $(O)$ cells were added to decreasing numbers of mesencephalic cells, the total cell concentration being constant $\left(5 \times 10^{5}\right.$ cells/dish). The mean length of DA neurites was estimated on the basis of the number of intersections between the neurites and a micrometer. About 100 neurons were measured in each case.
Influence of the target cell concentration on the mean length of DA neurites. In order to test whether neurite length correlates with the probability of encountering a target cell, we varied the concentration of striatal cells in the co-cultures. Decreasing concentrations of mesencephalic cells were co-cultured with increasing concentrations of striatal or cerebellar cells, maintaining a constant final cell density. From the results illustrated in Figure 7, the following conclusions can be made: (1) increasing the ratio of striatal cells to mesencephalic cells leads to a progressive decrease in the mean length of DA neurites; (2) replacing striatal cells by cerebellar cells at any concentration does not modify the mean length of DA neurites; (3) DA neurite elongation is independent of the number of mesencephalic DA or nonDA cells present. This latter observation confirms that these neurons in their majority have no effect on DA neurite elongation.

\section{Discussion}

Some of the morphological characteristics of mesencephalic DA neurons grown in primary cultures can be quantitatively analyzed following visualization of these cells by autoradiography after selective uptake of $\left[{ }^{3} \mathrm{H}\right]$ dopamine. Since the striatum is devoid of DA neuronal cell bodies, mesencephalic DA neurons can be distinguished in co-culture experiments and examined at the level of single identified neurons interacting with (some) other cells. The total lengths of DA processes including "dendrites" and the "axon" with their eventual collaterals were estimated either by direct measurement or by 
counting the number of intersections of the neurites with a micrometer. Using these approaches we were able to demonstrate that the presence of striatal target cells affects in vitro DA neurite elongation. Thus, a significant reduction in the total length of DA neurites is observed when DA neurons are cultured with striatal cells but not with the predominant mesencephalic non-DA cell population nor with cerebellar cells at any concentration tested. We suggest that the reduced length of DA neurites seen in the presence of striatal cells is linked to a direct and specific recognition between the growing tip of the DA neurites and the striatal target neurons. This conclusion is further supported by the absence of an effect on growth of the DA neurites when the neurons are cultured either with pure glial cells or in media conditioned with pure glial or neuronal cells. Therefore, direct contact with glial cells or diffusible factors which could be synthesized by either cell type (neuronal or glial) does not seem to play a role in the phenomenon observed in coculture with striatal cells. That direct neuro-neuronal interactions between DA neurons and striatal cells regulate the development or maturation of the afferent DA cells is in agreement with earlier conclusions reached on the hasis of biochemical data (Di Porzio et al., 1980). It is also supported by images showing interactions between DA terminals and identified neurons (BSP-2-positive cells, or cells cultured in serum-free conditions which virtually exclude the presence of glial elements). Complete demonstration of neuro-neuronal interactions between DA cells and their target will require ultrastructural investigations, presently in progress.

Our results raise two questions: (1) how does recognition operate, and (2) how does our in vitro system relate to the in vivo situation?

Numerous demonstrations of recognition of target have been obtained in vitro. Neurites outgrowing from explants of chick sensory ganglia in co-culture with ventral and dorsal horn fragments of the spinal cord systematically avoid the non-target ventral horn, yet they freely invade and establish functional synapses in the dorsal horn fragments (Peterson and Crain, 1982). A specific recognition was also shown by Bonhoeffer and Huf $(1980,1982)$ between the growth cones of retinal ganglia neurons and monolayers of tectum cells. Motor neurons can recognize through contact different regions of the extracellular matrix (Sanes et al., 1978), and peripheral as well as central neurons can distinguish between different substrate pathways (Katz et al., 1980).

Specific surface labels could be involved in a recognition process as described by Sperry (1963). However, antigens that are only expressed in specific neuronal populations have not yet been found. According to Barondes' hypothesis (1982), recognition could lead to stable adhesion through the externalization of intracellular ligands. Such cell adhesion molecules have been identified in many systems (Hausman and Moscona, 1976; Brackenbury et al., 1977; Trisler et al., 1981; Grumet et al., 1982). The role of differential adhesion in specifying nerve connections has been postulated by Fraser (1980) and has been studied in several experimental situations (Letourneau, 1975, 1979; Collins, 1980; Collins and Garret, 1980; Halfter et al., 1981). In all cases a correlation between strong adhesion and enhanced neuronal elongation has been observed. We can therefore imagine that there is a difference in adhesion strength between the DA growth cone and the surface offered by striatal neurons, on the one hand, and other cell types or polyornithine, on the other hand. Immunological and selective adhesion experiments are necessary in order to clarify this point.

Our results can be compared in some ways with numerous graft and regeneration experiments which have brought interesting information on the role of the environment in the cessation of neurite growth. For example, experimental designs have allowed to show that neurites grow for distances far greater than those observed in the normal physiological situation. This is the case of the retinal ganglion neurons of Xenopus whose lengths are several times the normal value when the eye primordia are transplanted at the tail level (Katz and Lasek, 1978). Similarly, axons from central neurons of the rat, when allowed to invade columns of Schwann cells, grow for distances far exceeding their normal length (Benfey and Aguayo, 1982). It can therefore be proposed that unique characteristics of specific target areas must provide signals which limit further outgrowth. It has been suggested that synaptic contacts with targets provide a continuous inhibition of axonal growth; this could be explained by reciprocal exchange of molecules, altered adhesivity, or localized disruption of cytoskeletal elements required for growth cone extension (Lasek and Black, 1977). Nevertheless, Scott and Muller (1980) have shown in regeneration experiments made in the leech that contact with the target, per se, is not required to interrupt axonal growth, but regenerating axons are stopped by environmental cues.

The various in vivo and in vitro experiments mentioned above strongly suggest that the choice made by the growing neurite to invade specific regions reflects specific interactions between the pre- and postsynaptic elements. However, in most cases, if not all, these experiments deal with grafts, explants, or confluent monolayers and do not allow us to examine the interactions taking place between one identified afferent fiber and one or a few target cells. Such a situation is achieved in our in vitro system in which dissociated cells are plated at low density. Under such conditions, the neurons are separated from each other by vast surfaces covered with polyornithine. Yet, growing axons seem to specifically recognize striatal cells, pause, and proceed until another striatal cell is encountered. This situation obviously differs from the much more complex one occurring in vivo since target cells are in close vicinity in an environment where growing axons are most likely guided by a combination of factors such as positional information, diffusible stimuli, and surface recognition. However, the end results of the two situations exhibit striking similarities. DA axons invade the whole target territory recognized as a terminal. It remains to be seen whether the elementary interaction between one DA fiber and one or more target cells is the key event in the interactions taking place in the highly specific process of innervation in vivo. It would also be interesting to know the identity of the target cells in vivo and in vitro. 
In previous work, it was shown that co-culturing mesencephalic DA neurons with striatal target neurons (Prochiantz et al., 1979; Di Porzio et al., 1980) or with membrane preparations from mouse striatum (Prochiantz et al., 1981) results in increased uptake and synthesis of dopamine demonstrating that target cells can influence biochemical maturation of the afferent neurons. In the present report we have demonstrated a second aspect of target influence upon afferent neurons, the ability of target neurons to limit the growth of their innervating neurons. It will be of considerable interest to continue with our dissection of the factors regulating DA neuronal growth in order to gain an understanding of the specific roles played by specific parts of the environment in the developing DA nigrostriatal pathway.

\section{References}

Barondes, S. H. (1982) Antibodies for studies of neuronal adhesion and recognition. Trends Neurosci. 5: 280-282.

Benfey, M., and A. J. Aguayo (1982) Extensive elongation of axons from rat brain into peripheral nerve grafts. Nature 296: 150-152.

Berger, B., U. Di Porzio, M. C. Daguet, M. Gay, A. Vigny, J. Glowinski, and A. Prochiantz (1982) Long-term development of mesencephalic dopaminergic neurons of mouse embryos in dissociated primary cultures: Morphological and histochemical characteristics. Neuroscience 7: 193-205.

Bonhoeffer, F., and J. Huf (1980) Recognition of cell types by axonal growth cones in vitro. Nature 288: 162-164.

Bonhoeffer, F., and J. Huf (1982) In vitro experiments on axone guidance demonstrating an antero-posterior gradient on the tectum. EMBO J. 1: 427-431.

Brackenbury, R., J. P. Thiery, U. Rutishauser, and G. M. Edelman (1977) Adhesion among neural cells of the chick embryo. I. An immunological assay for molecules involved in cell-cell binding. J. Biol. Chem. 252: 6835-6840.

Chuong, C. -M., D. A. McClain, P. Streit, and G. M. Edelman (1982) Neural cell adhesion molecules in rodent brains isolated by monoclonal antibodies with cross-species reactivity. Proc. Natl. Acad. Sci. U. S. A. 79: 4234-4238.

Collins, F. (1980) Neurite outgrowth induced by the substrate associated material from non-neuronal cells. Dev. Biol. 79: 247-252.

Collins, F., and J. E. Garrett, Jr. (1980) Elongating nerve fibers are guided by a pathway of material released from embryonic non-neural cells. Proc. Natl. Acad. Sci. U. S. A. 77: 62266228.

Di Porzio, U., M. C. Daguet, J. Glowinski, and A. Prochiantz (1980) Effect of striatal cells on in vitro maturation of mesencephalic dopaminergic neurons grown in serum-free conditions. Nature 288: 370-373.

Fraser, D. E. (1980) A differential adhesion approach to the patterning of cell connections. Dev. Biol. 79: 453-454.

Grumet, M., U. Ruthishauser, and G. M. Edelman (1982) Neural cell adhesion molecule is on embryonic muscle cells and mediates adhesion to nerve cell in vitro. Nature 295:
$693-695$.

Halfter, W., M. Claviez, and U. Schwartz (1981) Preferential adhesion of tectal membranes to anterior embryonic chick retina ncurites. Nature 292: 6770 .

Hausman, R. E., and A. A. Moscona (1976) Isolation of retinaspecific cell-aggregating factor from membranes of embryonic neural retina tissue. Proc. Natl. Acad. Sci. U. S. A. 73: 3594-3598.

Hirn, M., M. Pierres, H. Deagostini-Bazin, M. Hirsch, and C. Goridis (1981) Monoclonal antibody against cell surface glycoprotein of neurons. Brain Res. 214: 434-440.

Katz, M. J., and R. J. Lasek (1978) Eyes transplanted to tadpole tails send axons rostrally in two spinal-cord tracts. Science 199: 202-204.

Katz, M. J., R. J. Lasek, and H. J. W. Nauta (1980) Ontogeny of substrate pathway and the origin of the neural circuit pattern. Neuroscience 5: 821-833.

Lasek, R. J., and M. M. Black (1977) How do axons stop growing? Some clues for the metabolism of the proteins in the slow component of axonal transmort. In Mechanisms, Regulation and Special Functions of Protein Synthesis in the Brain, S. Roberts, A. Lajtha, and W. H. Gispen, eds., pp. 161-169, Elsevier/North Holland Biomedical Press, Amsterdam.

Letourneau, P. C. (1975) Possible roles for cell-to-substratum adhesion in neuronal morphogenesis. Dev. Biol. 44: 77-91.

Letourneau, P. C. (1979) Cell-substratum adhesion of neurite growth cones and its role in neurite elongation. Exp. Cell Res. 124: 127-138.

Peterson, E. R., and S. M. Crain (1982) Preferential growth of neurites from isolated fetal mouse dorsal root ganglia in relation to specific regions of co-cultured spinal cord explants. Dev. Brain Res. 2: 341-362.

Prochiantz, A., U. Di Porzio, A. Kato, B. Berger, and J. Glowinski (1979) In vitro maturation of mesencephalic dopaminergic neurons from mouse embryos is enhanced in presence of their striatal target cells. Proc. Natl. Acad. Sci. U. S. A. 76: 5387-5391.

Prochiantz, A., M. C. Daguet, A. Herbet, and J. Glowinski (1981) Specific stimulation of in vitro maturation of mesencephalic dopaminergic neurons by striatal membranes. $\mathrm{Na}$ ture 293: 570572 .

Prochiantz, A., M. C. Daguet, A. Delacourte, and D. Paulin (1982) Intermediate filament proteins in mouse brain cells cultured in the presence or absence of fetal calf serum. Exp. Cell Res. 139: 404-410.

Sancs, J. R., L. M. Marshall, and U. J. McMahan (1978) Reinnervation of muscle fiber basal lamina after removal of myofibers. J. Cell Biol. 78: 176-198.

Scott, S. A., and K. J. Muller (1980) Synapse regeneration and signals for directed axonal growth in the central nervous system of the leech. Dev. Biol. 80: 345-363.

Sperry, R. W. (1963) Chemoaffinity in the orderly growth of nerve fiber patterns and connections. Proc. Natl. Acad. Sci. U. S. A. 50: 703-707.

Trisler, D., M. D. Schneider, and M. Nirenberg (1981) A topographic gradient of molecules in retina can be used to identify neuron position Proc. Natl. Acad. Sci. U. S. A. 78: $2145-2149$. 